\title{
ATUAÇÃO DA RADIOFREQUÊNCIA NA GORDURA LOCALIZADA NO ABDÔMEN: revisão de literatura
}

Gabriele Morais LOFEU1

Karoline BARTOLOMEI ${ }^{2}$

Larissa Raquel Agostinho de BRITO

Alexandra Azevedo CARVALHO

1Pós graduada em Estética pela UninCor, gabi_lofeu@hotmail.com

2Pós graduada em Estética pela UninCor, kakazinhabdb@hotmail.com

3Pós graduada em Estética pela UninCor, larissabrito2@ hotmail.com

${ }^{4}$ Orientadora e professora Ms. no curso de graduação e pós graduação de Estética e Cosmética/ UninCor

Recebido em: 29/01/2015 - Aprovado em: 15/06/2015 - Disponibilizado em: 15/07/2015

\begin{abstract}
RESUMO
A exigência de silhueta corporal dentro dos padrões de beleza vigentes vem aumentando com o passar dos anos. Homens e mulheres desejam um corpo livre de imperfeições, e a gordura localizada vem sendo um dos maiores motivos de procura de tratamento em centros estéticos, academias, spa's e nutricionistas. A Radiofrequência é uma técnica utilizada principalmente para o estimulo e produção do colágeno, mas também gera uma energia que faz com que o aparelho alcance as camadas de gordura, reduzindo assim o volume das células gordurosas e acelerando o seu metabolismo, tornando-se um tratamento estético moderno e completo na perda de medidas abdominais. O principal objetivo desta pesquisa é revisar a literatura sobre a técnica de radiofrequência aplicada na gordura localizada no abdômen. Para estes fins, foram feitas diversas buscas em artigos científicos disponibilizados em bancos de dados científicos. Pesquisas atuais demonstram que o avanço tecnológico pode contribuir sobremaneira para melhorar disfunções estéticas, como é visto no uso da radiofrequência.
\end{abstract}

PALAVRAS- CHAVE: Radiofrequência. Abdômen. Gordura.

\begin{abstract}
The requirement of body silhouette within agreed standards of beauty has been increasing over the years. Men and women want a body free of imperfections, and localized fat has been one of the biggest reasons demand for treatment in beauty centers, gyms, spas and nutritionists. The Radio Frequency is a technique mainly used for stimulation and production of collagen, but also generates an energy that makes the machine reach the layers of fat, thereby reducing the volume of fat cells and speeding up your metabolism, making it a treatment modern and complete aesthetic in the loss of waist measurement. The main objective of this research is to review the literature on radiofrequency technique applied in localized fat in the abdomen. For these purposes, several searches were made in scientific articles available in scientific databases. A current research show that technological advances can contribute greatly to improve aesthetic disorders such as is seen in the use of radio frequency.
\end{abstract}

KEYWORDS: RF (radio frequency). Abdomen. Fat.

\section{INTRODUÇÃO}

Os tratamentos estéticos vêm ganhando uma grande importância no mundo contemporâneo, favorecendo a vida dos pacientes nos mais variados seguimentos da sociedade e fazendo com que o profissional desta área tenha maior importância entre as profissões da saúde e seja uma das que mais avança em termos de inovações, tanto de técnicas novas como de equipamentos eletro estéticos, como as radiações 
do espectro eletromagnético mais conhecido como radiofrequência (BORELLI, 2008).

Utilizada pela primeira vez no século XIX pelo físico francês Jacques Arsène D'Ansorval, a radiofrequência vem sendo utilizada até hoje nas práticas da dermatologia estética. A radiofrequência tornou-se um padrão de tratamento estético com muitas indicações, devido à sua versatilidade, eficácia e segurança. O conceito básico desta técnica não invasiva é a geração de calor no tecido subcutâneo, que induz a produção de novas fibras de colágeno e melhora o aspecto da pele. É uma corrente para tratamentos de linhas de expressão facial, fibroses recentes e tardias, cicatrizes e aderência, celulite, gordura localizada, contratura muscular, fibromialgia entre outras funções, como a contração das fibras de colágeno estimulando sua produção no tegumentar comum (BORGES, 2010).

Apesar de ser um recurso existente há mais de um século, no Brasil, apenas 2008 foi fabricada a primeira radiofrequência, chamada de Spectra ${ }^{\circledR}$ da empresa ToneDerm, seguido pelo equipamento Hertix ${ }^{\circledR}$ da empresa KLD para disfunções estéticas (AGNE, 2009) e logo depois fabricado pela empresa IBRAMED o aparelho Hooke com registro da ANVISA e com manutenção feita dentro da própria empresa.

\section{METODOLOGIA}

Este trabalho propôs-se a realizar uma revisão de literatura para avaliar o efeito da terapia de radiofrequência no tratamento de desordens do tecido adiposo que causam a gordura localizada. Esta pesquisa foi realizada através de levantamento bibliográfico em livros, artigos científicos e sites especializados sobre o assunto, com levantamento bibliográfico das publicações nas bases de dados do Scielo e Google Acadêmico através do uso os seguintes descritores: Radiofrequência, Gordura Localizada e Abdômen. Foram utilizados artigos de intervenção com a Radiofrequência, artigos complementares com o uso da Radiofrequência associado a outros aparelhos e cosméticos juntamente com pesquisas sobre gordura localizada sendo associada a outros tratamentos estéticos.

\section{RADIOFREQUÊNCIA}

(AGNE, 2009) Descreve: Trata-se de uma modalidade terapêutica que utiliza radiações do espectro eletromagnético na ordem de kilohertz (KHz), ou seja, radiofrequiência. Por ser uma onda senoidal de elevada frequência, perde seus efeitos químicos e biológicos de excitação neuromuscular, entretanto conserva o efeito de conversão em calor ao ser absorvida pelos tecidos. A

radiofrequência é uma onda eletromagnética que gera calor por conversão, compreendida entre $30 \mathrm{KHz}$ e $300 \mathrm{MHz}$, sendo a frequência mais utilizada entre 0,5 e $1,5 \mathrm{MHz}$. As correntes que se encontram abaixo do 3.000 Hertz (Hz) são empregadas na eletroestimulação e eletro analgesia. Em contrapartida a radiofrequência é utilizada na dermatologia para geração de calor por conversão. A conversão se refere à passagem da radiofrequência com comprimento de onda métrica e centimétrica pelo tecido do indivíduo que se converte em outra radiação, calor, cujo comprimento de onda está na ordem nanômetro. (CAPPONI, R. RONZIO, O. 2007) .

Por este processo é considerada genericamente como diatermia e é aplicada há anos como termoterapia profunda Nos últimos dez anos, variadas técnicas não invasivas e não cirúrgicas, mas que também se situam fora do âmbito médico da dermatologia, têm sido desenvolvidas para o tratamento do corpo em geral e da pele em particular. A tecnologia emergente que mais se tem 
destacado desde o tratamento com laser é a radiofrequência (ARNOCZKY, 2000).

Nas pesquisas foram utilizadas o aparelho Hooke produzido pela empresa IBRAMED que em seu manual descreve o aparelho da seguinte forma: "A radiofrequência é um aparelho com corrente alternada que promove diatermia, ou seja, o aquecimento através do calor profundo, onde converte a energia elétrica em energia térmica, promovendo um aquecimento em média de $40^{\circ}$ a $43^{\circ}$ graus no interior dos tecidos. $\mathrm{O}$ aparelho estudado Hooke possui três aplicadores para aplicação cutânea: dois aplicadores de radiofrequência: Bipolar (penetração superficial até $4 \mathrm{~mm}$ ) e monopolar (penetração profunda de 15 a 20 $\mathrm{mm})$ que produzem um campo eletromagnético de alta frequência (27,12 $\mathrm{MHz}$ ) e um aplicador criogênico e cooling (resfriamento eletrônico livre de água e livre de gás) para conforto térmico, resfriamento e preservação da epiderme que pode ser usado como pré-resfriamento e/ou pós-tratamento.

\section{GORDURA \\ LOCALIZADA ABDOMINAL}

O tecido adiposo é um órgão com varias funções: isolamento térmico, barreira física ao trauma, armazenamento energético e secreção de proteínas e peptídeos bioativos com ação local e à distância. (COSTA et al.,2006)

(GUIRRO \& GUIRRO, 2002) Explica: O tecido adiposo é uma forma de tecido conjuntivo, formado por células chamadas adipócitos. "Elas podem ser encontradas de forma isolada ou em pequenos grupos, nas malhas de muitos tecidos conjuntivos, ou ainda agrupadas em grandes áreas do corpo, como no tecido subcutâneo" O desenvolvimento irregular do tecido conjuntivo adiposo subcutâneo é popularmente conhecido como gordura localizada, podendo ser de origem genética ou produzida por alterações posturais ou circulatórias. Em pessoas de peso normal pode concentrar-se em aproximadamente de $15-20 \%$ do peso corporal em homens e em mulheres de $20-25 \%$.

De acordo com (FERREIRA, 2006) o tecido adiposo tem como função principal o armazenamento de energia em forma de triglicerídeos, suas células, os adipócitos, apresentam seu desenvolvimento a partir de células semelhantes aos fibroblastos, multiplicam-se durante a infância e adolescência, permanecendo um número constante durante a vida adulta. Sendo que no adulto pode variar a quantidade de lipídio depositado em seu interior.

De acordo com (GUIRRO, 2004), a má formação das células adiposas na infância é o principal motivo para a formação da adiposidade, mais entre esses fatores, destacase entre os principais predisponentes: genética, idade, sexo e desequilíbrio hormonal. Entre os fatores determinantes, os quais podem agravar os predisponentes, pode citar o estresse, o fumo, sedentarismo, maus hábitos alimentares e disfunções no organismo geral.

A gordura localizada apresenta-se como um desenvolvimento irregular do tecido conjuntivo subcutâneo. Neste caso, os adipócitos apresentam-se aumentados em regiões específicas com irregularidade do tecido e aparência ondulada. O processo de desenvolvimento de gordura corporal ocorre em razão do aumento no número de células adiposas, a hiperplasia celular; do aumento no volume de células já existentes, a hipertrofia celular; bem como da combinação destes dois fenômenos (GUIRRO \& GUIRRO, 2002).

Segundo (BORGES, 2006; GUIRRO e GUIRRO, 2002) o corpo humano possui 
capacidade limitada para armazenar carboidratos e proteínas, e a gordura contida no interior dos adipócitos representa o armazenamento de calorias nutricionais que excedem a utilização. Dessa forma, o tecido adiposo representa um reservatório de energia, principalmente em períodos de jejum prolongado, proteção contra frio ou quando o organismo está sujeito à atividade intensa.

Sendo assim, a gordura localizada possui suas funções até certa quantidade no corpo depois passa a ser prejudicial e incômoda quando questionada no ponto de vista estético. (BORGES, 2006; GARCIA et. al., 2006) Conclui que: $O$ excesso de tecido adiposo pode desencadear sérios problemas de saúde, pois reduz a expectativa de vida pelo aumento do risco de desenvolvimento de doenças cardíacas coronarianas, hipertensão, diabetes, osteoartrite e certos tipos de câncer. Este excesso de gordura pode existir mesmo em pessoas que não possuem um peso elevado.

\section{EFEITOS RADIOFREQUÊNCIA DA GORDURA LOCALIZADA NO ABDÔMEN}

Para (HASSUN K.M et. al., 2008) a radiofrequência é um tratamento não invasivo, que leva ao melhor aporte circulatório e de nutrientes, hidratação tecidual, aumento da oxigenação, aceleração da eliminação de catabólitos, lipólise, contração do tecido conectivo promovendo a reorientação de fibras de colágeno e incremento na contagem destas fibras, aumento da espessura e na densidade do tecido epitelial bem como a regeneração de tecidos moles, sendo indicada para pacientes com flacidez cutânea leve a moderada, para a melhora do contorno facial e corporal, atenuação de sulcos e rítides, retração moderada da área sub mentoniana e pescoço e tratamento da lipodistrofia ginóide, como todo equipamento de termo terapia profunda, causa uma excitação celular grande, levando a um gasto calórico acentuado, ao tempo que estaremos promovendo uma melhora expressiva da gordura localizada ou celulite na parte circulatória promovendo uma vasodilatação importante, porém quando há uma fibrose muito acentuada, recomenda-se inicialmente um pré-aquecimento rápido com a radiofrequência.

(BORGES, 2010) Ainda cita: "Para isso a radiofrequência, que é um aparelho que promove calor profundo, gera aquecimento no interior dos tecidos sendo responsável pela lipólise dos adipócitos, que possivelmente implicam em redução de medidas e reorganização das fibras de colágeno. Após o aquecimento observa-se a hiperemia da pele como consequência da vasodilatação e aumento do fluxo de sangue, que como efeito aumenta a circulação periférica e assim melhora a oxigenação do tecido por meio da corrente sanguínea".

(BORGES, 2010), Conclui que no combate a gordura localizada, o tratamento por radiofrequência permite o aumento da microcirculação sanguínea, a atividade enzimática, metabólica e térmica, ativando a "queima" da gordura, além de aumentar o consumo de energia em nível celular (ATP). As ondas de calor profundo produzidas pela radiofrequência possuem a capacidade de gerar energia e forte calor sobre a camada mais profunda da pele, enquanto a superfície se mantém resfriada e protegida, o que causa a contração do colágeno, além de ativar a produção de neocolágeno que desenvolve uma melhora ainda maior no aspecto da pele. Após o aquecimento observa-se a hiperemia 
da pele como consequência da vasodilatação e aumento do fluxo de sangue, que como efeito aumenta a circulação periférica e assim melhora a oxigenação do tecido por meio da corrente sanguínea.

$\mathrm{Na}$ década passada questionou-se a via metabólica da gordura hidrolisada. Hoje essa via já esta bem estudada. Para (ENJOJI, M. et. al., 2012) o adipócito é composto basicamente de colesterol e triglicérides na proporção de $20 \%$ de colesterol e $80 \%$ de triglicérides. Os triglicérides são compostos de ácidos graxos e glicerol. Após a cavitação, parte do conteúdo do adipócito entra em contato com as enzimas do liquido intersticial, sendo metabolizada. $\mathrm{O}$ ácido graxo que surge após a lipólise se liga a albumina, ganha a circulação sanguínea e caminha até o fígado, onde é eliminado pela bile. Já o glicerol, que é hidrossolúvel, se dissolve no plasma sendo posteriormente eliminado pelo fígado. $\mathrm{O}$ colesterol presente no organismo pode ter destinos diferentes. Para ser transportado no sangue e esterificado a uma molécula de ácidos graxos para aumentar sua hidrofobicidade e depois envoltos por uma lipoproteína. Explicando assim como é feita a eliminação do conteúdo da célula adiposa após o tratamento.

\section{APLICAÇÃO RADIOFREQUÊNCIA NA GORDURA ABDOMINAL}

Para (AGNE, 2009) e (MEYER, P. F. RONZIO, 2010) O efeito Joule é o principal efeito térmico da radiofrequência ao atravessar o organismo efetuando a produção de calor. Do efeito térmico ocorre outro efeito que é a vasodilatação periférica local. Devido ao calor gerado, consegue-se um aumento do fluxo sanguíneo e, portanto se produz uma melhora do trofismo, da oxigenação e do metabolismo celular. $\mathrm{O}$ aquecimento gerado por este equipamento promove a quebra dos tecidos adiposo e fibroso, além de melhorar a circulação sanguínea no local, que por sua vez, ajuda na drenagem de fluidos e toxinas. Outro benefício do aumento da temperatura é promove a restauração de colágeno, atenuando rugas (linhas de expressão) e flacidez tissular (BORELLI, 2008).

(GOMÉZ A.C., 2007) A energia gerada pela radiofrequência penetra em nível celular na epiderme, derme e hipoderme e alcança inclusive as células musculares. Quando passa pelos tecidos, a corrente gera uma ligeira fricção ou resistência dos tecidos, produzindo uma elevação térmica da temperatura tissular. No momento em que o organismo detecta uma maior temperatura que o fisiológico, aumenta a vasodilatação com abertura dos capilares, o que melhora o trofismo tissular, a reabsorção dos líquidos intercelulares excessivos e o aumento da circulação. Com isso, há um ganho nutricional de oxigênio, nutrientes e oligoelementos para o tecido, e também ocorre uma melhora no sistema de drenagem dos resíduos celulares (toxinas e radicais livres). Esses efeitos proporcionam a possibilidade de fortalecer a qualidade dos adipócitos, provocando lipólise homeostática e produção de fibras elásticas de melhor qualidade, atuando nos fibroblastos e em outras células. Logo, o tratamento por radiofrequência permite a elevação da temperatura interna do tecido promove aumento da circulação sanguínea em direção ao tecido conectivo, estimula a drenagem linfática, diminuindo a concentração de toxinas no adipócito, reduzindo assim seu tamanho, melhorando inclusive a gordura 
localizada, sendo um forte aliado em pósoperatório por agir diretamente nas fibroses.

De acordo com as recomendações e funcionalidade do manual, os efeitos do aparelho vendido pela empresa IBRAMED com registro da ANVISA Hooke cita: A radiofrequência e sua atuação na pele, na celulite e na gordura localizada a médio e longo prazo são: Inicialmente promove a contração imediata do colágeno; logo em seguida, ocorre a ativação de uma cascata de sinalização envolvendo mediadores do processo inflamatório e de reparação/regeneração tecidual, em resposta à leve lesão térmica promovida. Esse efeito promove a reestruturação da pele e do tecido subcutâneo, proporcionando uma aparência mais lisa e firme da mesma, através da deposição e remodelação do colágeno (neocolagênese) e da elastina (neoelastogenese), tendendo a perdurar e a incrementar o resultado ao longo dos meses.

Durante o procedimento o inicio é simples e indolor, um óleo de vegetal é aplicado para que a ponteira do aparelho deslize com facilidade ao longo da pele, aquecendo-a e massageando-a. O tratamento não é doloroso, dando apenas uma sensação de queimação da pele, e é interrompido quando a mesma chega a 40 graus, evitando, assim, a ocorrência de queimaduras. Para (GUIRRO \& GUIRRO, 2004), a radiofrequência consiste em ondas eletromagnéticas que provocam oscilação das moléculas de água, transformando energia eletromagnética em energia térmica e é o calor atuando na camada mais profunda da pele que causa a contração do colágeno. No caso de celulite e gordura localizada, o calor gerado pela radiofrequência aumenta a circulação e drenagem de fluidos, quebra o tecido adiposo e a fibrose.

\section{INDICAÇÕES, CONTRA INDICAÇÕES E RISCOS.}

O uso da radiofrequência está cada vez mais em evidência no meio estético para tratamentos de flacidez de pele, rugas, rejuvenescimento, celulite e contorno corporal. Trata-se de uma técnica não invasiva que através de correntes elétricas causam o aquecimento do tecido, induzindo a neocolagênese (crescimento do novo colágeno). Esta nova tecnologia não invasiva, é uma corrente usada para tratamentos de linhas de expressão facial, fibroses recentes e tardias, cicatrizes e aderência, celulite, gordura localizada, contratura muscular, fibromialgia entre outras funções, como a contração das fibras de colágeno estimulando sua produção no tegumentar comum (BORGES, 2010).

A radiofrequência é indicada em todos os processos degenerativos que impliquem na diminuição ou retardo do metabolismo, irrigação e nutrição, sendo em geral em patologias crônicas. Também é indicado por provocar aumento da vasodilatação e irrigação abaixo da zona tratada, além da oxigenação e nutrição dos tecidos (RODRIGUEZ, J. M. Martín, 2004).

Existem algumas contra indicações para o uso da radiofrequência que são: alterações na sensibilidade do paciente, utilização de metais no corpo, implantes elétricos, gestantes, pacientes em tratamentos com medicamentos para a circulação sanguínea, utilização sobre glândulas hormonais, hemofílicos, focos de infecções e indivíduos com febre. Qualquer aparelho eletrônico ou metais devem ser retirados próximo do aparelho de radiofrequência durante a aplicação da técnica (CARVALHO et al. 2011). 
É contra indicado o uso da radiofrequência em indivíduos com transtorno de sensibilidade, com o uso de metais intraorgânicos, osteossínteses, implantes elétricos, marca-passo, sobre glândulas que provoquem aumento de hormônio, grávidas, em focos infecciosos, pacientes que estejam ingerindo vasodilatadores ou anticoagulante, hemofílicos e em indivíduos com processos febris. É recomendado não aplicar simultaneamente com outros aparelhos de eletroterapia e também retirar correntes, aparelhos eletrônicos e elementos metálicos de perto do aparelho (RODRIGUEZ, J.M. Martín, 2004) e (RONZIO, O. A.2006)

$\mathrm{Na}$ aplicação dos transdutores, temos que tomar algumas precauções iniciais como verificar a integridade da pele no local a ser tratado (se houverem lesões não devemos aplicar), com o aplicador sempre em movimento em uma das mãos e na outra o termômetro digital para aferir qual o aumento de calor na região aplicada. Em média, na medição da temperatura superficial, atingimos 36 a 38 graus Celsius, ${ }^{2,4}$ o que corresponderá internamente na região tratada, uma temperatura 3 a 4 graus Celsius acima da temperatura superficial. (BORGES, 2010) e (ALVAREZ, 2008). Porém, jamais devemos nos esquecer que o parâmetro absoluto é a tolerância do paciente e toda e qualquer referência dolorosa deverá ser seguida por diminuição da intensidade aplicada ou até a cessação da aplicação.

\section{DISCUSSÃ̃}

A radiofrequência é indicada em todos os processos degenerativos que impliquem na diminuição ou retardo do metabolismo, irrigação e nutrição, sendo em geral em patologias crônicas. Também é indicado por provocar aumento da vasodilatação e irrigação abaixo da zona tratada, além da oxigenação e nutrição dos tecidos. A energia de radiofrequência é uma forma de energia eletromagnética, segura, eficaz e não ablativa (Atua principalmente nas camadas médias e profundas da pele, causando pouco ou nenhum dano na camada mais superficial) que pode ser aplicada a qualquer tipo de pele (RODRIGUEZ, 2004). Quando aplicada à pele, criam-se campos eletromagnéticos oscilantes que provocam o movimento dos elétrons nos tecidos, e a corrente eléctrica resultante gera um calor interno proporcional à resistência eléctrica da pele. As moléculas de colágeno são produzidas pelos fibroblastos e este, quando aquecido, sofre uma transição estrutural, transformando-se numa estrutura semelhante a um gel e, como tal, menos organizada (ARNOCZKY, 2000).

De acordo com (BORGES, 2010), a vasodilatação e a hiperemia surgem como consequência do efeito térmico, em que a vasodilatação promove um aumento da circulação periférica local, gerando a hiperemia na pele. Assim como no efeito térmico, a hiperemia apenas ocorre com o uso de intensidade alta, por um tempo maior de aplicação, portanto este efeito não é verificado. A oxigenação celular está ligada à vasodilatação e ao consequente aumento do fluxo sanguíneo, aumentando desta forma, o aporte de oxigênio por intermédio da corrente sanguínea.

Para (BORGES, 2010), (ALVAREZ, 2004) e (WANITPHAKDEEDECHA, 2006) Conclui que a gordura localizada no tratamento de radio frequência, como todo equipamento de termo terapia profundo sofre uma excitação celular grande, levando a um gasto calórico acentuado, ao tempo que estaremos promovendo uma melhora expressiva da gordura localizada ou celulite 
na parte circulatória promovendo uma vasodilatação importante.

$\mathrm{O}$ estudo realizado mostra um tratamento amplo e completo, que ajuda prevenir a flacidez durante o emagrecimento. Para (AGNE, 2009), A radiofrequência consiste na emissão de uma onda de rádio que atua em profundidade, fazendo aquecer os tecidos de sustentação da pele, a hipoderme e a gordura subcutânea. Uma ação que permite não só promover a síntese do colágeno, levando à criação de neocolágeno, como também modificar as células de gordura, contraindoas. Desta forma, a pele ganha firmeza e o corpo fica remodelado. Ela promove uma contração imediata das fibras de colágeno e a formação destes elementos, atuando também, no aumento do metabolismo e na diminuição da gordura e na melhoria das fibras.

Juntamente com o tratamento proposto de gordura localizada, a pele também passa a sofrer uma atuação do colágeno. Segundo (BORGES, 2010) e (ALVAREZ, 2008) Também menciona a atuação na perda de medida e produção do colágeno. "O colágeno é uma proteína que inicia seu processo de desnaturação a partir de 45 graus Celsius e nosso intuito nesta modalidade de tratamento é causar uma retração no mesmo, fazendo com que haja uma nova estruturação tecidual que diminuirá a flacidez tecidual, principalmente o que faz o tratamento moderno e completo, beneficiando o paciente também no futuro."

(GEDDS, 1997) Cita: Quando aplicada na pele, a corrente promove na derme aquecimento que estimula a circulação sanguínea através do calor e auxilia na formação de novas fibras colágenas além de reestruturar as fibras antigas, o que contribui para espessamento e firmeza da pele. Para (HARRIS, 2005), conclui-se que o efeito térmico da radiofrequência no tecido causa alterações ocasionadas igualmente a um processo inflamatório, que nada mais é que uma reação do organismo à um processo agressor, sendo assim, o organismo age da mesma forma obtendo as fases do processo inflamatório. Resumidamente, o uso da radiofrequência por esteticistas é indicado para alterações causadas nas fibras de colágeno, que através da energia térmica causa o aquecimento tecidual e induz a neocolagênese. Mostrando assim a eficácia do tratamento também na prevenção da flacidez quando o paciente emagrece.

\section{CONSIDERAÇÕES FINAIS}

A aplicação da radiofrequência mostrouse promissora para a área estética, obtendo resultados positivos. O principal benefício pode ser concluído que além de melhorar o contorno corporal irregular causado pela gordura localizada, ele beneficia melhorando o colágeno no local, evitando a flacidez da pele com a redução de medidas, tornando o tratamento completo e moderno na área estética.

(GOMÉZ, 2007), a energia penetra em nível celular em epiderme, derme e hipoderme e alcança inclusive as células musculares. Quando passa pelos tecidos, a corrente gera uma ligeira fricção ou resistência dos tecidos com passagem da radiofrequência, produzindo uma elevação térmica da temperatura tissular. No momento que o organismo detecta uma maior temperatura que o fisiológico, aumenta a vasodilatação com abertura dos capilares, o que melhora o trofismo tissular, a reabsorção dos líquidos intercelulares excessivos e o aumento da circulação. Com isso, ocorre um ganho nutricional de oxigênio, nutrientes e oligoelementos para o tecido, influenciado 
pela radiofrequência, com uma melhora no sistema de drenagem dos resíduos celulares (toxinas e radicais livres). Estes efeitos proporcionam a possibilidade de fortalecer a qualidade dos adipócitos, provocando lipólise homeostática e produção de fibras elásticas de melhor qualidade, atuando nos fibroblastos e em outras células.

O protocolo realizado por (COSTA et. al. 2009) mostra que a utilização da radiofrequência foi aplicada em humanos por 12 sessões, três vezes por semana, visando à redução da adiposidade abdominal. O tempo utilizado foi de três minutos por área e a quantidade de áreas de acordo com a superfície abdominal a ser tratada, determinado de acordo com estudo de (LOW e REED, 2001), correspondendo a duas áreas do eletrodo ativo, tempo necessário para chegar a valores com aumento de $5^{\circ}$ a $6^{\circ} \mathrm{C}$ (que teve como média inicial $33,7^{\circ} \mathrm{C}$ e média final $40,5^{\circ} \mathrm{C}$ avaliadas com termômetro de superfície), totalizando uma média de 20 minutos.

O procedimento é muito seguro. A ocorrência de pequenos danos à pele é raríssima. $\mathrm{O}$ tratamento foi estudado cuidadosamente em centenas de pacientes nos EUA e Canadá gerando relatórios que mostram mínimos efeitos adversos. A radiofrequência é utilizada no tratamento de diferentes alterações estéticas que dependem da reestruturação do colágeno, como no rejuvenescimento cutâneo e no remodelamento corporal, pois, além de atuar nas rugas e flacidez facial, também atua na fibroedema gelóide, além de obter o efeito reafirmante no corpo (CARVALHO et al. 2011). Levando a conclusão do tratamento completo e inovador no mercado estético.

(CARVALHO et al. 2011). Ainda conclui que: $O$ tempo da realização de análise de dados referente à radiofrequência não se deve limitar apenas à dados colhidos imediatamente após o tratamento, isso se deve ao fato de que o colágeno continua o processo de reestruturação até mesmo 6 meses após o estimulo térmico na temperatura adequada $\mathrm{E}$ (ZELICKSON et al., 2004) Também conclui que: A produção de neocolágeno irá ocorrer durante semanas após a aplicação da radiofrequência, gerando uma melhora no aspecto e qualidade da pele. Os efeitos biológicos da radiofrequência constituem no aumento da circulação arterial, vasodilatação, melhorando assim a oxigenação e a acidez dos tecidos aumento da drenagem venosa, aumentando a reabsorção de catabólitos e diminuindo edemas nas áreas com processos inflamatórios aumento da permeabilidade da membrana celular, permitindo uma melhor transferência de metabólitos através desta estimulação do sistema imunológico e diminuição dos radicais livres (CARVALHO, 2011).

A associação de terapias foi um fator em comum entre os estudos de revisão bibliográfica utilizados neste trabalho, foi citado nos estudos de (MONTESI et al., 2007) a associação da radiofrequência e da massagem mecânica no mesmo aparelho. Essa técnica de radiofrequência com massagem mecânica consiste em aumentar a circulação tecidual para que o efeito da radiofrequência tenha uma melhor penetração no tecido sendo utilizada de forma isolada ou associada a outro recurso como massagem modeladora, aparelhos como cellutec e vacuoterapia.

Os resultados encontrados mostram que a técnica é segura, efetiva e bem tolerada na questão de dor, tratando-se de procedimentos corporais não invasivos. $\mathrm{O}$ retorno às atividades normais do dia a dia é momentâneo e a técnica não invasiva atrai ainda mais a procura pelo tratamento. Essa técnica também 
seria bem empregada para pacientes não recomendados para o tratamento cirúrgico. Sempre bom reiterar que uma boa anamnese com histórico de doenças prévias acompanhada de exame físico detalhado e analise do nível de expectativa dos pacientes e fundamentais para o sucesso e também para evitar os transtornos resultantes da insatisfação dos pacientes. Complicações do aquecimento podem ocorrer, mas são raras. Não há cuidado especial que seja necessário após o tratamento é recomendado apenas o uso de protetor solar.

Todos os artigos selecionados tiveram o mesmo propósito de avaliar a radiofrequência

\section{REFERÊNCIAS BIBLIOGRÁFICAS}

AGNE, J. E. Eu sei eletroterapia. Santa Maria: Pallotti, 2009

ALVAREZ N, ORTIZ L, Vicente V, Alcaraz $M$, Pedreno $\mathrm{P}$ S. The effects of radiofrequency on skin: experimental study. Lasers in Surgery and Medicine. 2008;

ARNOCZKY SP, Aksan A. Thermal modification of connective tissues: basic cience considerations

and clinical implications. J Am Acad Orthop Surg ; 8: 305-13; 2000.

BORGES, Fábio dos Santos.

Dermatofuncional: modalidades terapêuticas nas disfunções estéticas. São Paulo: Phorte, 2006.

BORGES, F. S. Modalidades terapêuticas nas disfunções estéticas. 2. Ed. São Paulo: Phorte, 2010

BORELLI, S. S. Até 120 anos rejuvenescimento e cosmético. São Paulo: Senac, 2008 em técnicas não invasivas nas diferentes aplicações em estética. Alguns autores também relataram que os resultados foram inferiores aos obtidos em procedimentos invasivos tais como toxina botulínica e cirurgias, foi observado que a associação da massagem mecânica e manual, aumenta a penetração da radiofrequência. Apesar do pequeno número de artigos publicados selecionados no intervalo de tempo estipulado pelo presente estudo, os trabalhos analisados apresentaram resultados promissores já que todos os artigos consultados obtiveram melhora do aspecto estético após intervenção.

CARVALHO, G. F.; SILVA, R. M. V.; et AL: Evaluation of the radiofrequency effects on connective tissue. Especial Dermatologia, vol. 68, pag. 10-25, 2011.

CAPPONI, R. RONZIO, O. Manual de Fisioterapia. Cap. XIV Argentina Maimónides 2007 (In press).

COSTA JV, DUARTE JS - Tecido adiposo e adipocinas. Acta Med Port 2006;

COSTA, E.M. DANTAS, J.S.C. Furtado, F.N. Medeiros, M.L., Meyer, P.F. Ronzio, O.A. Avaliação dos efeitos do uso da tecaterapia na adiposidade abdominal. Kinesia, V. 1, n. 1, Mar, 2009.

DICIONÁRIO Houaiss de Física: Onda de rádio / Radiofrequência. Editora Objetiva, vol. 1, pag. 162-192, 2005.

ENJOJI M, Yasutake K, Kohjima M, Nakamuta M. Nutrition and nonalcoholic Fatty liver disease: the significance of cholesterol. Int J Hepatol. 2012

FERREIRA, E.C.et al. Análise da cicatrização da bexiga com o uso de extrato aquoso da Orbignya phalerata (babaçu): estudo controlado em ratos. Act Cir. Bras. 2006, 
V.21, suppl.3, p.33-39. ISSN 0120-8650. Disponível em: Acesso em 14 de junho de 2010.

GARCIA, P. G.; GARCIA, F. G.;BORGES, F. S.; O uso da eletrolipólise na correção da assimetria no contorno corporal póslipoaspiração: Relato de caso. Revista Fisioterapia Ser - Ano 1 - nr 4 -out/nov/dez 2006

GOMÉZ, A.C. Radiofrequência capacitiva em Celulitis. Casuística. Anais do XVI Congresso Mundial de Medicina Estética. Argentina: Buenos Aires, Abril 11-14, 2007.

GEDDS, S.; DEWITT, D.P. O que há de novo em instrumentação eletrocirurgico e instrumentação medico. [s.1.]: Ed boca raton, 1997. v. 11.

GUIRRO, E.C.O.; GUIRRO, R.R.J. Fisioterapia Dermato-Funcional:

Fundamentos, Recursos e Patologias. 3. ed. São Paulo: Manole, 2002, p.437-447.

GUIRRO, E.; GUIRRO, R. Fisioterapia Dermato-Funcional: Fundamentos - Recursos - Patologias. 3 ed. Barueri: Manole, 2004.

HARRIS, M. I. N. C.: Pele - Estrutura propriedades e envelhecimento. $2^{\mathrm{a}}$ edição. Editora Senac. São Paulo, pag. 121-189, 2005.

HASSUN K.M; BAGATIN E; VENTURA K. F. Radiofrequência e Infravermelho. Rev. Bras. Med. 2008, 65(n.esp):18-20.

LOW, J. Reed, A. Eletroterapia explicada: princípios e práticas. 3. ed. São Paulo:

Manole, 2001.
Manual do aparelho de Radiofrequência Hooke, disponível em: www.ibramed.com.br/wpcontent/files_mf/1363703256Hooke.pdf acesso em 27/01/2015

MEYER, P. F. RONZIO, O. A. Radiofrequência. In: BORGES, F. S. Fisioterapia Dermato-Funcional: Modalidades Terapêuticas nas Disfunções Estéticas. São Paulo: Phorte, 2010 Cap. 25, p.601-620.

MONTESI G, CALVIERI S, BALZANI A, GOLD MH.: Bipolar radiofrequency in the treatment

of dermatologic imperfections:clinicopathological and immunohistochemical aspects. Journal of Drugs Dermatology, vol. 6, pag. 890-896, 2007.

RODRIGUEZ, J.M. Martín. Electroterapia em Fisioterapia.Rio de Janeiro: 2. ed. Panamericana, 2004.

RONZIO, O. A. Que es la Tecaterapia?. El Kinesiologo Argentina, 2006.

WANITPHAKDEEDECHA R, Manuskiatti W. Treatment of cellulite with a bipolar radiofrequency, infra red heat and pulsatile suction device:a pilot study. 2006. J CosmetDermatol;Dec;5(4):284-8.

ZELICKSON, B.D.; KIST D.; BERNISTEIN, E.; et al. Histological and Ultrastructural Evaluation of the Effects of a Radiofrequency-Based Nonablative Dermal Remodeling Device: A Pilot Study. Arch Dermatol. Vol. 140,pag. 204-209, 2004 